\title{
Depression Detection from Bangla Facebook Status using Machine Learning Approach
}

\author{
Masum Billah \\ Department of Computer science and Engineering \\ Sylhet Engineering College \\ Sylhet, Bangladesh
}

\author{
Enamul Hassan \\ Department of Computer science and Engineering \\ Shahjalal University of Science and Technology \\ Sylhet, Bangladesh
}

\begin{abstract}
Depression is a mood disorder. This mental illness is a silent killer. It badly affects how human feels, thinks, and acts. Every year, all over the world huge number of people commit suicide due to depression[1]. In Asia region, most of the Bengali speaking people use Facebook rather than Twitter for their social communication and share most of their emotions there. That is why Facebook is a potential source for our research. Although detecting depression is a psychological matter, in this paper there was an attempt to predict depression using machine learning. Here, a model is proposed to predict depressed or not depressed using machine learning from Bangla Facebook Status. The work is done with 50 Facebook users' data, among them 17 people had committed suicide. Naive Bayes[2], Multinomial Naive Bayes[3], Logistic Regression[4], Linear SVC[5] and some other classifiers used for this work. The work achieved satisfying accuracy as the first work in Bangla language.
\end{abstract}

\section{General Terms}

Machine learning, Supervised Learning, Bangla Facebook Status, Depression Detection, Sentiment Analysis.

\section{Keywords \\ Banglish.}

\section{INTRODUCTION}

Depression is a buzzword in modern life. According to WHO (World Health Organization), about 300 million people are affected from this hidden mental illness. Around 800000 people all around the world dies due to suicide every year being suffered from depression [6]. Depression can't be recognized easily like any other physical diseases. It needs psychologist's help to identify this. Suicide due to depression is increasing day by day. In china and Japan suicide rate for depression is very high. $70 \%$ of the patients would not consult doctors at early stages of depression [7]. Many people think that, depression belongs to only rich people. This is a very wrong concept. Even in developing country, like Bangladesh people suffer from depression and suicide. In Bangladesh, up to $80 \%$ of the people don't go to psychologists due to various reasons. Some people think, if they go to psychologists then people will laugh at them and their social status will be down. Eventually those depressed people become more depressed day by day, and after some period of time, some of them choose the way of suicide to make them free from depression. In recent year more than 20 incidents of suicidal occurrences have seen in Bangladesh, and most of them shared their last post on their Facebook profile. Among all of them one thing was common. That is, they all were very disappointed to their life before their death. If those depressed people could be identified before they reach lethal stage, then they might be saved from suicide. In traditional way depression is detected by taking interview and asking some question made by psychologists. When psychologists observe a depressed people, he notices some special side of his Behavior and many other factors. As most of the people usually don't go to psychologists, so computer power could be used to analyze peoples' emotional stage from their social profile.

In this modern era, most of the people are dependent on social media. They share their all feelings on social sites like Facebook, Twitter etc. They share all their feeling of happiness, sadness, anger, depression etc. So, now social media has become a potential source of analyzing emotions of people. Most of the Bengali people use Facebook rather than Twitter. For this reason, Facebook was chosen as the working field. Some of the related work have seen in Chinese, English and Spanish language. But no work in this topic was found in Bangla language.

In this paper, the aim was to build a model that can predict depressed or not depressed analyzing Facebook status. Every country has their own style of expressing emotions. Bengali peoples have their own linguistic style. At First, depressed peoples' Facebook status were analyzed and some features of their way of expressing depression came up from this analysis. After that, the dataset is developed based on those features. Traditional machine learning algorithms were the first choice along with various features, and the model is tested. As Bangla Facebook statuses are mixed with wrong spelling, incomplete words, meaningless punctuations, English words and Banglish words (Bangla interspersed with English, a blend of Bangla and English), all of these things made this work even harder.

\section{PREVIOUS WORKS}

Depression detection is one kind of sentiment analysis. Some works has been done on this topic. Some of them are discussed below.

\subsection{A Depression Detection Model Based on Sentiment Analysis in Micro-blog Social Network}

[8] Developed a model for depression detection from twitter using sentiment analysis. At first they proposed a sentiment analysis method using vocabulary, Linguistic rules. After that they develop a model based on proposed method and they used 10 features extracted from depressed peoples who were in observation of psychologist. In their work they used three kind of classifier and their accuracy is $80 \%$. Their developed model analyzes users' interaction with others, User behaviors in micro-blog, The use of emoticons and The use of first person singular and plural pronouns for detecting depression. But they used only dataset from 180 twitter users. And their model work on Chinese language. 


\subsection{The Psychology of Word Use in Depression Forums in English and in Spanish: Testing Two Text Analytic Approaches}

[9] done their research work for proving that, the online depressed writers use more first-person singular pronouns but less first-person plural pronouns, more negative emotion words but less positive emotion words for this they collect posts from Internet forums of depressed and non-depressed people they observed linguistic markers of depression on those posts. They analyze the text with LIWC, this is a word counting tool for their work. They observed linguistic markers of depression in English and Spanish forums using 160 depressed and 160 non-depressed people's posts. They also analyzed the themes; people use when talking about their depression in forms in English and Spanish through 404 English and 400 Spanish depressed people.

\subsection{Predicting Depression via Social Media}

Here in [10], researchers proposed a method for Predicting Depression via Social Media. For this work, they collect 100 people twitter status of last year, who was being diagnosed with clinical depression. They also collect 476 twitter users' tweets as training data purpose. By observing the depressed people, they find out behavioral attributes relating to social engagement, emotion, language and linguistic styles, ego network. After that they build a classifier (SVM) including those features for predicting likelihood of being depressed. With their recognized features their classifier gave $70 \%$ accuracy. Their proposed method can only predict from English tweets.

\subsection{Detecting Suicidal Ideation in Chinese Micro-blogs with Psychological Lexicons}

[11] Proposed their method for detection of Suicidal Ideation in Chinese Micro-blogs using psychological lexicon dictionary. At first they explore linguistic features and made a classifier based on those features. They used Naive Bayes, Logistic Regression, J48, Random Forest, SMO, SVM algorithms. Their accuracy for detection of suicide ideation is $79 \%$, and overall the accurate reach $94 \%$. For dataset they collect 53 verified suicidal users with 30000 posts, 600000 posts from 1000 thousand random non-suicidal users. But they identified incorrectly $40 \%$ of suicidal posts. And their work on only for English language posts.

\subsection{Improving the Accuracy of Suicide Attempter Classification}

For [12] research 849 cases are acquired from hospital. They scored them manually by psychological standards or questionnaires then those scores were used to evaluate four classifiers, including Boosting, Linear Discriminate analysis, Fisher linear discriminant analysis and Support Vector Machine (SVM). They noticed that the scores that they gave manually to the people can be used to identify suicidal person using classifiers.

\subsection{The language of paranoia.}

This [7] research work is done for proving that linguistic analysis of speech could classify patients into diagnostic groups such as those suffering depression and paranoia. Doing a survey on these papers, it is seen that, none of the papers proposed any system for detecting depression from Bangla textual data. All of the works were done on various languages, but in Bangla language, no work has been found on this topic.

\section{METHODOLOGY}

Bangla language is comparatively more complex to understand than English. That's why methods applied on English language won't work properly for Bangla language. As depression detection is mainly done by psychologists, so for this work, it is the crying need to contact psychologists for extracting features of depressed people. The proposed method is described below.

\subsection{Dataset creation}

Facebook statuses of individuals were collected by using own developed crawler where user tokens were used. With the help of Facebook Graph API user tokens were collected and then run the crawler for collecting Facebook statuses of individuals. Unfortunately, no Depression dataset in Bangla language were found. So, a self-developed dataset was needed and it was developed from the collected data. The developed dataset contains only Bangla status. Some small English words were kept which may contain more information. Some English statuses were also translated into Bangla for increasing data quantity. Any kind of status which is related to political issues, photo credit, Check-in, tagged post etc. were removed. Because, these types of status do not contain user's own emotions. Depression is identified by psychologists, but psychologists come up with some common linguistic features of depressed people. Depressed people use more first-person singular pronouns as "me", "myself" and "I", than second and third person pronouns. This means they focus more on themselves rather than others. They uses an excessive amount of words conveying negative emotions, specially negative adjectives and adverbs - such as "lonely", "sad" or "miserable" [13]. They also use more "Absolutist words" such as: "always", "nothing" or "completely" [13]. So, these types of status are added in the dataset. Some examples are given below.

Table 1. Sample Bangla Status

ফ্যামিলির জন্য এত কিছু করি তাও আমাকে তারা মানুষ বলেই গণ্য

করে না :'(

হঠাৎ করে পর হয়ে যাওয়া আপনজনকে অনেক দূর থেকে মিস করার

থেকে বেশি যন্ত্রণা আর কিছুতেই নাই

আমাকে কেউ বাঁচতে দিল না :( আল্লাহ হাফেজ

Our small dataset contains only 1000 status with Depressed or Not Depressed tag. Among them 800 status are used for training and 200 status is used for testing purpose.

\subsection{Data preprocessing}

As collected user data is very messy and noisy, the collected data were preprocessed. This task has been done in some steps such as punctuation removal, tokenization, stop words removal, normalization. The package NLTK was also be used for preprocessing task. From the raw Bangla Facebook status, at first, punctuations were removed. They are symbols, which are used in writings and creating sentences. Although punctuation makes sentence clearer but they are not significant in summarization process. All the punctuations were not removed. Rather a small list of punctuations, numbers and other unnecessary symbols which contains no subjective information were noted. Then those symbols were removed from the raw text. Punctuations which are used in writing emoticons are not removed. After removing 
punctuations, clean data is found. From there the text is tokenized. Stop words usually contain no meaning. Some of the common stop words in Bangla: এবং(And), অথবা(Or), কিন্তু(But). These words contain very less information. So, these are also removed to reduce complexity.

\section{With punctuations:}

হরর স্টোরি শুনবেন ??!..... সেমিস্টার ফাইনালের তারিখ দিয়ে দিসে :'( :'(

After removing punctuations:

হরর স্টোরি শুনবেন সেমিস্টার ফাইনালের তারিখ দিয়ে দিসে :'( :'(

Before tokenized:

মুক্তাগাছা ঘুরাঘুরির পর মুক্তগাছার বিখ্যাত মন্ডার দোকানের মন্ডা খাওয়া (:)

\section{After tokenized:}

<মুক্তাগাছা><ঘুরাঘুরির>>পর>>মুক্তগাছার><বিখ্যাত><মন্ডার><দোকানের >< মন্ডা|<খাওয়া><-;>>

\section{Some of the stop words are given below:}

উনি, উপর, অতএব, অথচ, অন্য, অবধি, এঁরা, এই, একই, আছে, আজ, একে, ই, ইত্যাদি, ইহা, এখানে, এখানেই, এটা, এতে, ওকে, করে,

কয়েক, চলে, জনকে, থেকে

\subsection{Normalization and Stemming}

Bangla sentences are full of misspellings and incomplete words. Same word may be seemed different for misspellings and incompleteness. Such as: অরথ (meaning) and অর্থ (meaning) both are the same word, but due to misspelling the same word is counted as two different words. Same problem arises if stemming is not done. That is why stemming was needed. Example of stemming is in table 2.

Table 2. Sample of Stemming

\begin{tabular}{|c|c|}
\hline Before Stemming & After Stemming \\
\hline মধ্যরাতের & মধ্যরাত \\
\hline খাইতেছিল & খাচ্ছিল \\
\hline আসতেছি & আসছি \\
\hline
\end{tabular}

Sometimes Emoticons contains more clear emotion than text. For this feature, 16 different types of Emoticons were kept. Emoticons are written with punctuations. Some Emoticons are given below:

[ --- ], [ :/ ], [ ;P ], [ :P ], [ :) ], [ :( ], [ ;) ], [^^^], [ <3 ], [ :O ], [ :-( ], [ :D ], [ :'( ], [ 3:) ], [ O:) ], [ B) ]

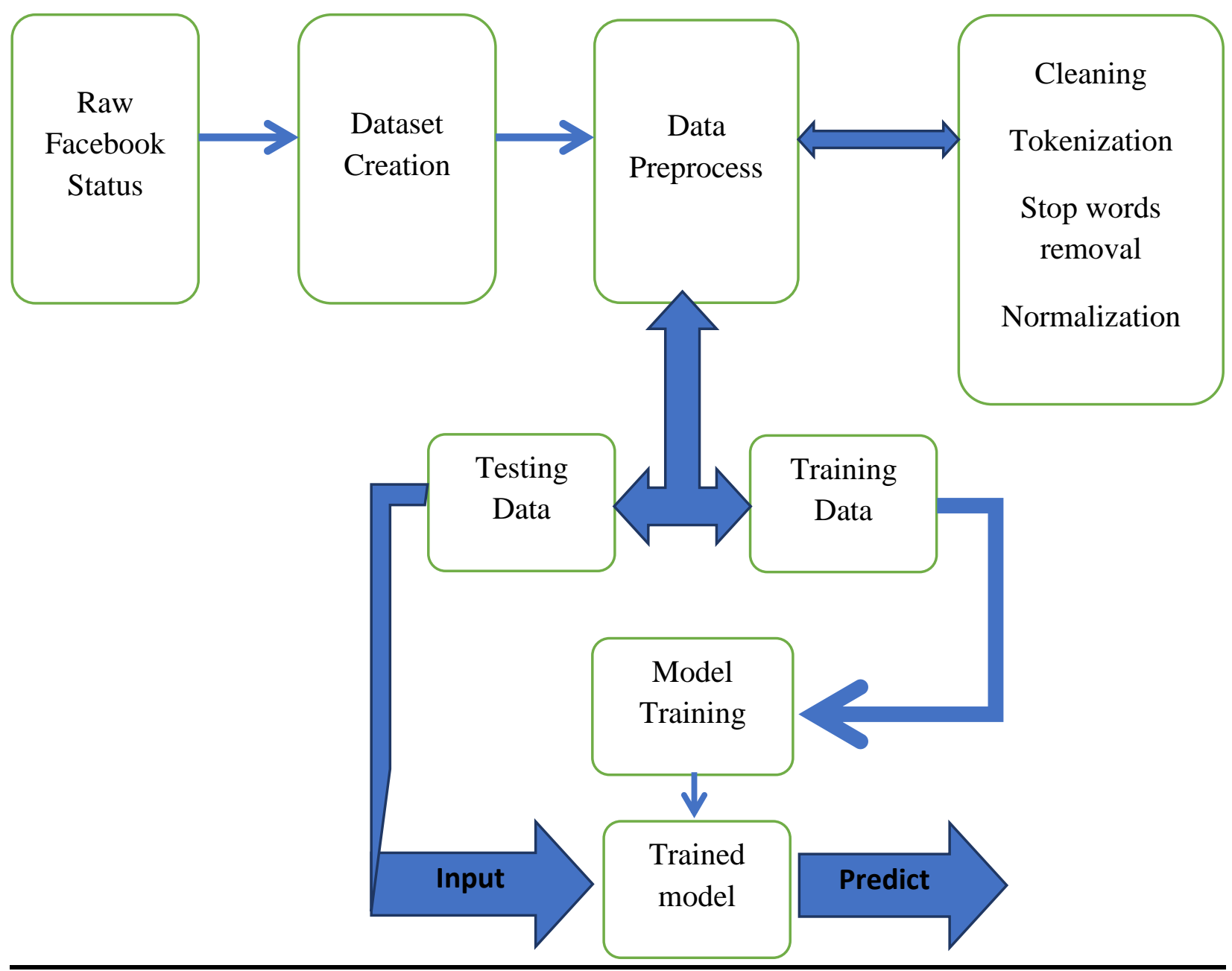

Figure 1. Diagram of proposed model 


\subsection{Extracting features}

In feature extraction, each tweet is represented as a set of features called a feature vector. The proposed model is tested for 5 different types of features.

1. Unigram.

2. Unigram and Emoticons.

3. Bigram and emoticons.

4. Unigram and Emoticons with TF-IDF values.

5. Unigram + emoticons as well as depressive words.

For each feature, BOW was created according to the feature. Then each status was converted into a feature vector based on the presence or absence of the terms in the BOW. An example of feature extraction is given in table 3 .

TF-IDF values of each term were also used instead of 'True' or 'False' value.

$$
\begin{gathered}
T F(w)=\frac{\# \text { of times term } w \text { appears in a document }}{\text { Total \# of terms in the document }} \\
I D F(w)=\ln \left(\frac{\text { Total } \# \text { of documents }}{\# \text { of documents with term } w \text { in it }}\right) \\
T F-I D F=(T F * I D F)
\end{gathered}
$$

\subsection{Classifiers}

When the full dataset is converted into feature vectors, the whole dataset were split into $80 \%$ and $20 \%$. $80 \%$ status were

\begin{tabular}{|c|c|}
\hline Sample status & আমার দুনিয়াটা অনেক ছোট :) \\
\hline Feature vector & $\begin{array}{l}\text { \{'আম্মু': False, 'মারা': False, } \\
\text { 'যাওয়ার': False, 'আমার': } \\
\text { True, 'দুনিয়াটা': True, } \\
\text { 'ছোট': True, 'হয়ে': False, } \\
\text { 'গিয়েছিল': False, :): True }\end{array}$ \\
\hline
\end{tabular}
used for training purpose and $20 \%$ for testing the accuracy.

Table 3. Example of Feature Vector.

NLTK and Scikit-learn algorithms were used. Scikit-learn is a library of Python that provides many unsupervised and supervised learning algorithms. Original Naive Bayes, Multinomial Naive Bayes, Bernoulli Naive Bayes, Logistic Regression, Linear Support Vector Classifier, Number Support Vector Classifier were used from Scikit-learn.

\subsection{Experimental Results and Evaluation}

There are some ways for measuring accuracy. In this work, F-

\begin{tabular}{|c|c|c|c|c|c|c|c|c|c|c|}
\hline & \multicolumn{2}{|c|}{$\begin{array}{c}\text { Multinomial Naïve } \\
\text { Bayes }\end{array}$} & \multicolumn{2}{|c|}{ SGDC classifier } & \multicolumn{2}{|c|}{ Logistic Regression } & \multicolumn{2}{|c|}{ Linear SVC } & \multicolumn{2}{|c|}{$\mathrm{Nu} \mathrm{SVC}$} \\
\hline & $\begin{array}{c}\text { Depresse } \\
\text { d F- } \\
\text { measure }\end{array}$ & $\begin{array}{c}\text { Not } \\
\text { Depresse } \\
\text { d F- } \\
\text { measure }\end{array}$ & $\begin{array}{l}\text { Depresse } \\
\text { d F- } \\
\text { measure }\end{array}$ & $\begin{array}{c}\text { Not } \\
\text { Depresse } \\
\text { d F- } \\
\text { measure }\end{array}$ & $\begin{array}{c}\text { Depresse } \\
\text { d F- } \\
\text { measure }\end{array}$ & $\begin{array}{c}\text { Not } \\
\text { Depresse } \\
\text { d F- } \\
\text { measure }\end{array}$ & $\begin{array}{c}\text { Depresse } \\
\text { d F- } \\
\text { measure }\end{array}$ & $\begin{array}{c}\text { Not } \\
\text { Depresse } \\
\text { d F- } \\
\text { measure }\end{array}$ & $\begin{array}{l}\text { Depresse } \\
\text { d F- } \\
\text { measure }\end{array}$ & $\begin{array}{c}\text { Not } \\
\text { Depresse } \\
\text { d F- } \\
\text { measure }\end{array}$ \\
\hline Unigram & 0.64 & 0.72 & 0.64 & 0.75 & 0.65 & 0.77 & 0.65 & 0.76 & 0.65 & 0.76 \\
\hline $\begin{array}{c}\text { Unigram } \\
+ \\
\text { Emoticon } \\
\mathrm{s}\end{array}$ & 0.73 & 0.61 & 0.73 & 0.77 & 0.75 & 0.75 & 0.74 & 0.73 & 0.74 & 0.72 \\
\hline $\begin{array}{l}\text { Bigram + } \\
\text { Emoticon } \\
\mathrm{s}\end{array}$ & 0.55 & 0.71 & 0.55 & 0.71 & 0.55 & 0.71 & 0.55 & 0.71 & 0.55 & 0.71 \\
\hline $\begin{array}{c}\text { Unigram } \\
+ \\
\text { Emoticon } \\
\text { s with } \\
\text { TF-IDF } \\
\text { value }\end{array}$ & 0.71 & 0.72 & 0.69 & 0.73 & 0.71 & 0.73 & 0.71 & 0.72 & 0.71 & 0.71 \\
\hline $\begin{array}{c}\text { Unigram } \\
+ \\
\text { Emoticon } \\
\mathrm{s}+ \\
\text { Depresse } \\
\mathrm{d} \text { words } \\
\text { list }\end{array}$ & 0.72 & 0.65 & 0.71 & 0.73 & 0.72 & 0.73 & 0.72 & 0.73 & 0.71 & 0.73 \\
\hline
\end{tabular}
measure is used for calculating accuracy.

Table 4. F-measure for various types of algorithms 
Table 5. Accuracy (in \%) of algorithms along with various features

\begin{tabular}{|c|c|c|c|c|c|}
\hline & Unigram & $\begin{array}{c}\text { Unigram + } \\
\text { Emoticons }\end{array}$ & $\begin{array}{c}\text { Bigram + } \\
\text { Emoticons }\end{array}$ & $\begin{array}{c}\text { Unigram + } \\
\text { Emoticons with } \\
\text { TF-IDF values }\end{array}$ & $\begin{array}{c}\text { Unigram + Emoticons + } \\
\text { Depressive words list }\end{array}$ \\
\hline Multinomial Naïve Bayes & 70.99 & 62.71 & 55.93 & 74.78 & 71.18 \\
\hline SGDC classifier & 67.17 & $\mathbf{7 7 . 9 6}$ & 55.93 & 70.43 & 72.88 \\
\hline Logistic Regression & $\mathbf{7 1 . 7 5}$ & $\mathbf{7 4 . 5 7}$ & 55.93 & 73.04 & 72.88 \\
\hline Linear SVC & 71.75 & 71.18 & 55.93 & $\mathbf{7 7 . 3 9}$ & 72.88 \\
\hline Nu SVC & 70.99 & 69.49 & 55.93 & 74.78 & 78 \\
\hline
\end{tabular}

True Positive (tp): is defined as the number of tweets, from the test set, correctly labeled by the classifier as belonging to a particular class or label.

True Negative (tn): is defined as the number of tweets, from the test set, correctly labeled by the classifier as not belonging to a particular class or label.

False Positive (fp): is defined as the number of tweets, from the test set, incorrectly labeled by the classifier as belonging to a particular class or label.

False Negative (fn): is defined as the number of tweets, from the test set, that are not labeled by the classifier as belonging to a particular class or label but should have been.

F-measure is the weighted harmonic mean of precision and recall for a particular class. That is,

$$
\mathrm{F}-\text { measure }=\frac{2 * \mathrm{P} * \mathrm{R}}{\mathrm{P}+\mathrm{R}}
$$

Multinomial Naive Bayes, SGDC classifier, Logistic Regression, Linear SVC and NuSVC classifier with 5 types of features are used.

From the Table 4, Using Unigram feature, the highest Fmeasure value is found for depressed class 0.65 and not depressed class 0.76 from Linear SVC and Nu SVC. Overall accuracy of both algorithms is $71.75 \%$ (From the Table 5).

$$
\begin{aligned}
& \operatorname{Precision}(P)=\frac{\mathrm{tp}}{\mathrm{tp}+\mathrm{fp}} \\
& \operatorname{Recall}(R)=\frac{\mathrm{tp}}{\mathrm{tp}+\mathrm{fn}}
\end{aligned}
$$

Again, using unigram + emoticons, it is seen that SGDC classifier provides the highest F-measure 0.73 for depressed class and 0.77 for not depressed class. Over all accuracy is $77.96 \%$ (From the Table 2). Here accuracy is increased by $6.21 \%$ for using Emoticons.

For better result, bigrams and emoticons are used, but unfortunately this feature did not provide better result for our analysis. After that, unigrams + emoticons were used with their TF-IDF values. Linear SVC provided $77.39 \%$ accurate result with this feature.

At last, the model is tested with unigrams + emoticons + Depressive words as features. This feature showed less accuracy than previous feature. With this feature SGDC provided $74.57 \%$ accuracy.

But satisfying equal F-measure was found for both classes using Logistic Regression. The F-measure value for Logistic Regression is 0.75 , using unigrams + emoticons as feature.
Overall accuracy of Logistic Regression is $74.57 \%$ (From the table 5). Figure 2 shows overall accuracy for all used algorithms. The performance of all used algorithms could easily be compared from there. Specific accuracy were shown for each specific feature in the work in table 5. It could be observed that best results are found for (Unigram + Emoticons) and (Unigram + Emoticons with TF-IDF values) feature using SGDC classifier, Logistic Regression, Linear SVC. The Experimental result shows that, Emoticons and Unigrams is the best feature for finding the best result.

As no work found in Bangla language on this topic, so this work is compared with previous work, which are done in different language. Researchers of [8] used 10 features and 3 different classifiers. Their work is done on Chinese language and their accuracy is $80 \%$. On the other hand, [11] analyzed behavioral attributes relating to social engagement, emotion, language and linguistic styles, ego network. After that they develop a classifier based on those features and got $70 \%$ accurate result. But in this work 5 features and 5 different algorithms were used.

But, due to lack of Facebook Data our model provided best $77.96 \%$ accurate result. As the first attempt in Bangla, this result is not so bad.

\section{CONCLUSION AND FUTURE WORK}

In this paper, only attempt was to predict depressed or not depressed analyzing Facebook status. As this is the first work in Bangla, so the model is developed based on only social media status. But there are some factors that are not brought in consideration in this paper. But analyzing them, this model will be able to detect depressed people more accurately in social media. The next work of the continuation of this work is to enhance the current work. In present work, only 50 Facebook users were taken into consideration. The model is developed and tested with various features. The highest 77.96 $\%$ accuracy was found for SGDC with unigram + emoticons as feature. In this paper, only traditional machine learning procedures were used due to lack of data, but in future, the work would be to build the model using neural network for better accuracy. If the accuracy reach near $100 \%$, an attempt to deploy this on online could be done for depression detection. After detecting depressed people on social media, online counseling service would be offered for them where free online counseling will be provided to keep safe people from this dangerous mental illness. 


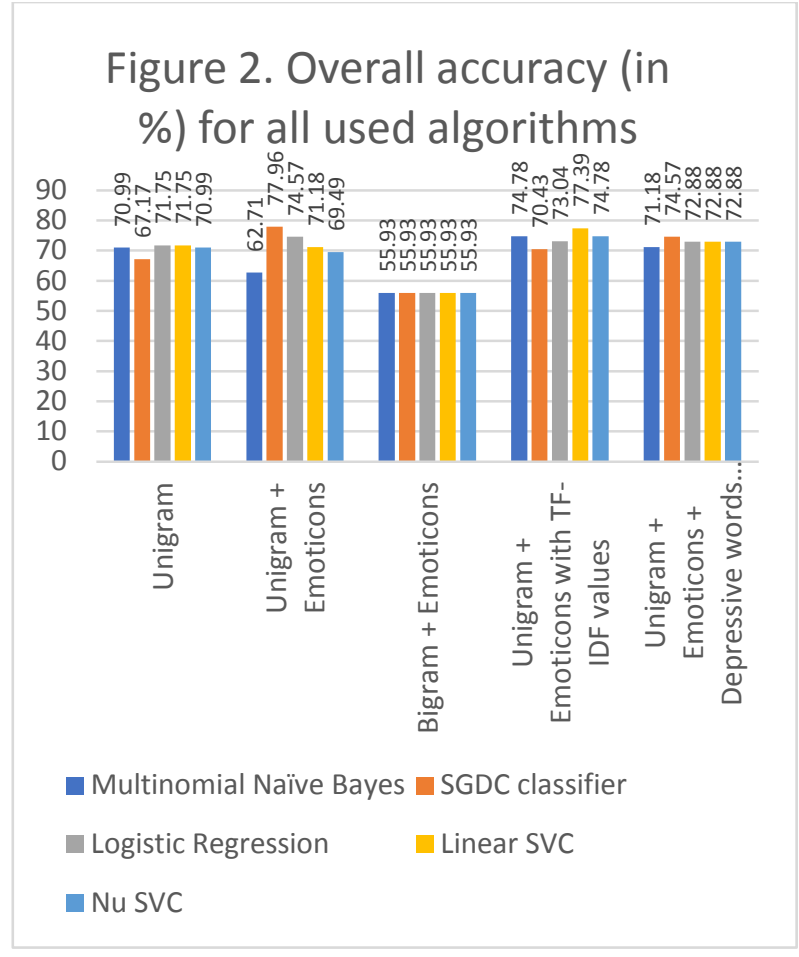

\section{ACKNOWLEDGMENTS}

To do a good research work, resources are very important thing. For this research, credit goes to Shahjalal University of Science and Technology as well as its affiliated college named Sylhet Engineering College. More specifically, a big thanks goes to the department of Computer Science and Engineering of both of the institutions for supervising and supporting this research work.

\section{REFERENCES}

[1] Gürhan, N., Beşer, N.G., Polat, Ü. and Koç, M., 2019. Suicide Risk and Depression in Individuals with Chronic Illness. Community mental health journal, 55(5), pp.840848.

[2] Rish, I., 2001, August. An empirical study of the naive Bayes classifier. In IJCAI 2001 workshop on empirical methods in artificial intelligence (Vol. 3, No. 22, pp. 4146).

[3] Kibriya, A.M., Frank, E., Pfahringer, B. and Holmes, G., 2004, December. Multinomial naive bayes for text categorization revisited. In Australasian Joint Conference on Artificial Intelligence (pp. 488-499). Springer, Berlin, Heidelberg.
[4] Hosmer Jr, D.W., Lemeshow, S. and Sturdivant, R.X., 2013. Applied logistic regression (Vol. 398). John Wiley \& Sons.

[5] Fan, R.E., Chang, K.W., Hsieh, C.J., Wang, X.R. and Lin, C.J., 2008. LIBLINEAR: A library for large linear classification. Journal of machine learning research, 9(Aug), pp.1871-1874.

[6] World Health Organization. 2018. Depression. Available Online at https://www.who.int/news-room/factsheets/detail/depression

[7] Oxman, T.E., Rosenberg, S.D. and Tucker, G.J., 1982. The language of paranoia. The American journal of psychiatry.

[8] Wang, X., Zhang, C., Ji, Y., Sun, L., Wu, L. and Bao, Z., 2013, April. A depression detection model based on sentiment analysis in micro-blog social network. In Pacific-Asia Conference on Knowledge Discovery and Data Mining (pp. 201-213). Springer, Berlin, Heidelberg.

[9] Ramirez-Esparza, N., Chung, C.K., Kacewicz, E. and Pennebaker, J.W., 2008, March. The Psychology of Word Use in Depression Forums in English and in Spanish: Texting Two Text Analytic Approaches. In ICWSM.

[10] De Choudhury, M., Gamon, M., Counts, S. and Horvitz, E., 2013, June. Predicting depression via social media. In Seventh international AAAI conference on weblogs and social media.

[11] Huang, X., Zhang, L., Chiu, D., Liu, T., Li, X. and Zhu, T., 2014, December. Detecting suicidal ideation in Chinese microblogs with psychological lexicons. In 2014 IEEE 11th Intl Conf on Ubiquitous Intelligence and Computing and 2014 IEEE 11th Intl Conf on Autonomic and Trusted Computing and 2014 IEEE 14th Intl Conf on Scalable Computing and Communications and Its Associated Workshops (pp. 844-849). IEEE.

[12] Delgado-Gomez, D., Blasco-Fontecilla, H., Alegria, A.A., Legido-Gil, T., Artes-Rodriguez, A. and BacaGarcia, E., 2011. Improving the accuracy of suicide attempter classification. Artificial intelligence in medicine, 52(3), pp.165-168.

[13] Al-Mosaiwi, M. and Johnstone, T., 2018. In an absolute state: Elevated use of absolutist words is a marker specific to anxiety, depression, and suicidal ideation. Clinical Psychological Science, 6(4), pp.529-542. 